\title{
Simple fabrication of Ag nanoparticle-impregnated electrospun nanofibres as SERS substrates
}

\author{
ALTANGEREL AMARJARGAL ${ }^{1,2, *}$, LEONARD D TIJING ${ }^{3,4}$ and CHEOL SANG KIM ${ }^{1,4}$ \\ ${ }^{1}$ Department of Bionanosystem Engineering, Chonbuk National University, Jeonju, Jeonbuk 561-756, Korea \\ ${ }^{2}$ Power Engineering School, Mongolian University of Science and Technology, Ulaanbaatar, Mongolia \\ ${ }^{3}$ School of Civil and Environmental Engineering, University of Technology, Sydney, Broadway NSW 2007, Australia \\ ${ }^{4}$ Division of Mechanical Design Engineering, Chonbuk National University, Jeonju, Jeonbuk 561-756, Korea
}

MS received 14 October 2013; revised 28 January 2014

\begin{abstract}
A facile method for the fabrication of electrospun polyurethane (PU) nanofibres impregnated with Ag nanoparticles (NPs) as an efficient and free-standing surface-enhanced Raman scattering (SERS) substrates is reported here. Electrospinning was used to produce polymeric nanofibrous matrix, while a liquid polyol(ethylene glycol) solvent under low temperature was used not only to reduce $\mathrm{Ag}^{+}$to $\mathrm{Ag}^{\mathbf{0}}$, but also was employed as the in situ growth medium for well-dispersed Ag NPs on the surface of fibre nets. Large enhancement in Raman signals of 4-mercaptobenzoic acid analytes could be realized in the present Ag/PU nanofibres due to the presence of SERS 'hotspots' by means of appropriate interparticle gap.
\end{abstract}

Keywords. Ag nanoparticles; electrospun nanofibres; polyol; SERS.

\section{Introduction}

In the last decade, surface-enhanced Raman scattering (SERS) has grown to become a powerful analytical tool for ultrasensitive detection of chemical and biological molecules down to the single molecular level, which can be applied in various fields including life science, analytical chemistry, biomedical and environmental science. ${ }^{1-7}$ SERS offers tremendous enhancement of the Raman signals by molecules in the presence of a nanostructured metallic surface, especially noble metals such as $\mathrm{Ag}$, $\mathrm{Au}$ and $\mathrm{Cu}$ nanoparticles (NPs). In general, it is widely accepted that the enhancement effect of SERS usually proceed via two distinct mechanisms: electromagnetic enhancement that is associated with the localized surface plasmon resonances occurring at the surfaces of roughened metal substrates or metal clusters and chemical enhancement that is associated with the direct charge transfer or indirect electron-hole pair excitation processes. ${ }^{8,9}$

Recent studies have found that the substrates having remarkable SERS activity/sensitivity can be achieved by controlled assembly or deposition of noble metal NPs on/in the one-dimensional (1D) nanostructure due to the presence of Raman 'hotspots' by means of gap distance between particles. When deposited plasmonic NPs on the support are in close proximity, individual plasmon oscillations can couple with each other, resulting in huge local electromagnetic field enhancements in these confined junctions or these

\footnotetext{
*Author for correspondence (a_amaraa1 @yahoo.com)
}

hotspots. ${ }^{10-13}$ For example, Zhang et $a l^{13}$ fabricated flexible and highly active/sensitive SERS substrates by electroless plating of Ag NPs on the surface of electrospun nanofibres via the seed-mediated growth process and suggested that the density and size of those deposited Ag NPs which to be controlled by experimental parameters led to the appropriate interparticle gap, suitable for the generation of Raman 'hotspots'. Furthermore, Bao et al $^{14}$ fabricated poly(methyl methacrylate) (PMMA) nanofibres in the form of overlaid films, surface decorated with Ag NPs facilitated via oxygen plasma etching, which were explored as active substrates for SERS.

Here, we report a simple and economical way to massproduce $\mathrm{Ag}$ /polyurethane (PU) nanofibres. The aim of the present work was to prepare efficient and free-standing SERS substrates with high enhancement factor (EF) by electrospun nanofibres impregnated with Ag NPs through combining the methods of electrospinning and polyol immersion, in the absence of any surfactant or sensitizing and stabilizing reagent. Here, the function of ethylene glycol solvent under low temperature was not only to reduce $\mathrm{Ag}^{+}$ to $\mathrm{Ag}^{0}$, but also was employed as the in situ growth medium for well-dispersed Ag NPs on the surface of fibre nets.

\section{Materials and methods}

\subsection{Electrospinning}

Polyurethane (10 wt $\%$ ) solution was prepared by dissolving appropriate amount of PU pellets (Skythane ${ }^{\circledR}$ X595A-11) in $N, N$ dimethylformamide (DMF) and methyl ethyl ketone/2butanone (MEK) (50/50, wt:wt\%, Showa) solvent solution. 

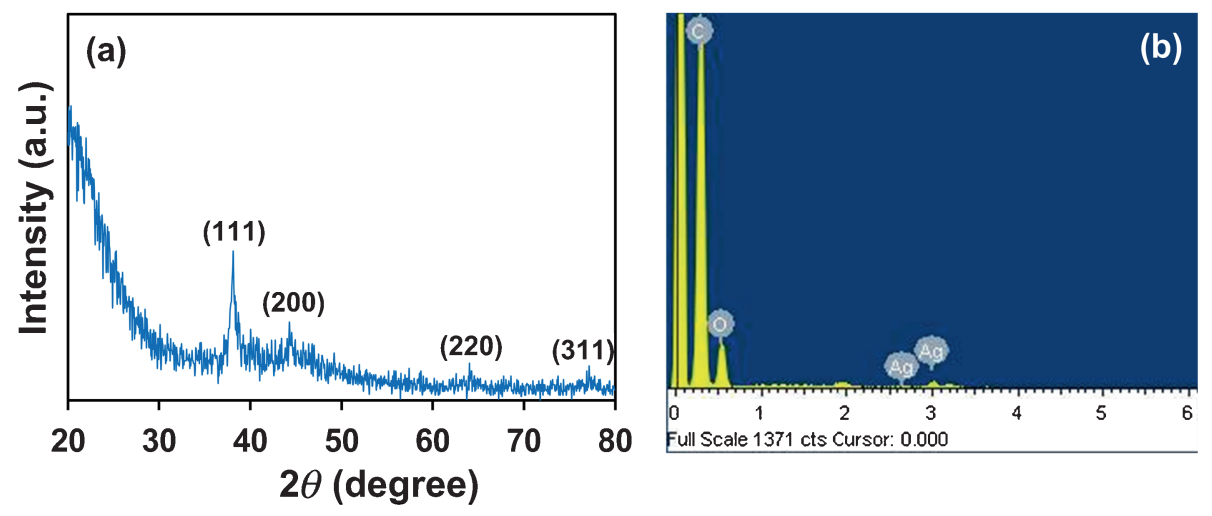

Figure 1. (a) XRD pattern and (b) EDS spectrum of the Ag NP-impregnated electrospun PU nanofibres.

Electrospinning was carried out at $11 \mathrm{kV}$, tip-to-collector distance of $15 \mathrm{~cm}$ and solution feed rate of $1 \mathrm{ml} \mathrm{h}^{-1} \cdot{ }^{15}$ During electrospinning, the nozzle (inner diameter $=0.51 \mathrm{~mm}$ ) kept on moving laterally (i.e., back and forth) on its axis for a distance of $150 \mathrm{~mm}$ and a linear speed of $100 \mathrm{~mm} \mathrm{~min}^{-1}$ controlled by LabVIEW 9.0 (National Instruments). Four millilitres of PU solution was electrospun onto a grounded flat collector, which was perpendicularly oriented to the nozzle. After electrospinning, the PU nanofibrous mat was dried at $80^{\circ} \mathrm{C}$ for $48 \mathrm{~h}$ to remove the residual solvents.

\subsection{Fabrication of Ag-PU composite nanofibres}

The Ag NP-impregnated electrospun PU nanofibres were performed by the polyol immersion technique, similar to our previous report. ${ }^{16,17}$ Briefly, electrospun PU nanofibrous mats $(5 \mathrm{~cm} \times 5 \mathrm{~cm})$ were immersed in a previously prepared EG solution containing dissolved $\mathrm{AgNO}_{3}$ and PVP, incubated at $60^{\circ} \mathrm{C}$ under soft shaking. Mat samples were taken out after $20 \mathrm{~h}$ of immersion, and were then thoroughly rinsed with deionized water and dried at $60^{\circ} \mathrm{C}$ for $24 \mathrm{~h}$.

\subsection{Characterization}

The surface structure and morphology of the present sample powders were studied by field emission scanning electron microscopy (FE-SEM, S-7400, Hitachi, Japan), and the elemental composition was checked using an energy dispersive spectrometer (EDS). X-ray powder diffraction (XRD) analysis was carried out by a Rigaku X-ray diffractometer $(\mathrm{Cu} \mathrm{K} \alpha$, $\lambda=1.54059 \AA$ ) over Bragg angles ranging from $20^{\circ}$ to $80^{\circ}$. SERS measurements were performed using a Nanofinder 30 Raman confocal spectroscopy device (Tokyo Inst. Co., Japan) equipped with a He:Ne laser $(\lambda=633 \mathrm{~nm})$ and the scattering peaks were calibrated with a reference peak from a Si wafer $\left(520 \mathrm{~cm}^{-1}\right)$. A $\times 40$ microscope objective was used to focus the laser beam to obtain the SERS spectra, and the laser power incident on the samples was $2 \mathrm{~mW}$ excitation.

\section{Results and discussion}

By utilizing two-step route, the well-distributed assembly of Ag NPs on the electrospun nanofibres was successfully fabricated through a simple and controllable manner. The structure of the $\mathrm{Ag} / \mathrm{PU}$ composite nanofibres was characterized by XRD and it is shown in figure 1a. The number of welldefined Bragg reflections with $2 \theta$ values at $38.2^{\circ}, 44.6^{\circ}, 64.2^{\circ}$ and $77.2^{\circ}$ in the XRD pattern of $\mathrm{Ag} / \mathrm{PU}$ composite nanofibres can be indexed to (111), (200), (220) and (311) crystal faces of the fcc metallic silver, respectively. The successful impregnation of Ag NPs on the PU nanofibres was confirmed by EDS showing the presence of $\mathrm{Ag}$ peaks (figure 1b).

Figure 2 shows the representative FESEM images at low and high magnification for the PU nanofibre membranes before and after impregnated with Ag NPs. As shown in figure $2 \mathrm{a}$ and $\mathrm{a}^{\prime}$, the surface of neat PU nanofibres is relatively smooth and the ultrafine matrix of interlocking fibres with submicron sizes, high porosity and randomly ordered morphology was found. After immersion in polyol medium for $20 \mathrm{~h}$, the outer surfaces of PU nanofibres were significantly rough by means of impregnated with Ag nanoparticles (figure $2 b$ ). As can be clearly observed in figure $2 b^{\prime}$, the $\mathrm{Ag}$ NPs with narrow size distribution were uniformly decorated on the surface of nanofibres. Generally, in order to decorate or assemble metal NPs on surfaces of electrospun nanofibres, many studies reported on the use of an immersion technique followed by post-treatment in the presence of surfactant, sensitizing and stabilizing reagent. ${ }^{18-20}$ However, in the present study, the PU nanofibres have been uniformly decorated with well-dispersed Ag NPs without using any additional chemicals to modify the nanofibre surfaces, except for ethylene glycol dispersion medium. Here, the possible assembly mechanism of Ag NPs on the PU nanofibres could be explained on the basis of hydrogen bonding interactions between the amide groups along with the PU backbone and the hydroxyl groups capped on the surface of the Ag NPs. ${ }^{18}$ After immersion, the PU mat turned from white to yellow and maintained this colour even after several washing with water (see insets of figure $2 a$ and $b$ ). 

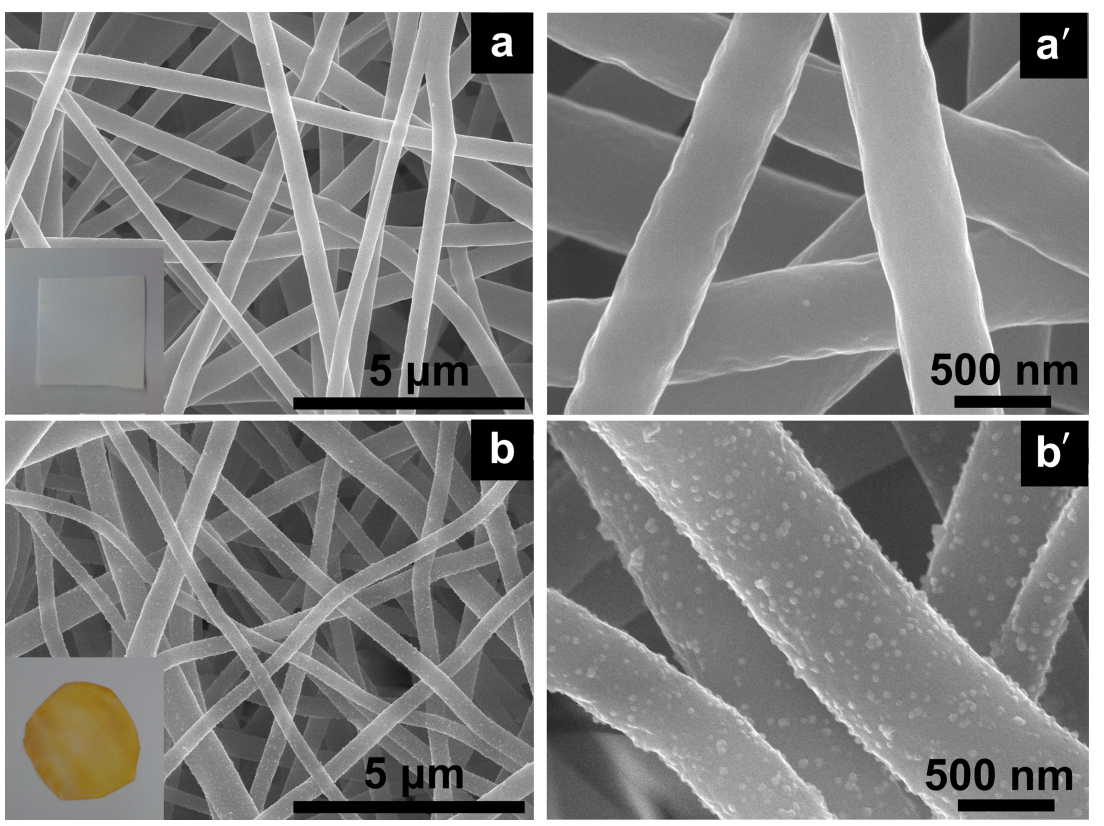

Figure 2. Low- and high-magnification FE-SEM images of the electrospun neat and composite nanofibres: (a and $\left.\mathbf{a}^{\prime}\right)$ PU and (b and $\mathbf{b}^{\prime}$ ) Ag/PU.

The uniformly distributed Ag NPs on the electrospun PU nanofibres can absorb analytes directly on their surfaces, leading to superior SERS sensitivity and they may provide a unique substrate for Raman applications. For this purpose, 4mercaptobenzoic acid (4-MBA) is used as a probe molecule to evaluate the SERS performance of the prepared nanostructures. As shown in figure 3, normal Raman scattering spectrum of 4-MBA powder as well as the SERS spectra from PU-Ag NPs nanofibrous membranes are comparable to those reported previously for 4-MBA adsorption. ${ }^{7,21}$ In particular, characteristic vibrational modes, including $v(\mathrm{CC})$ ring-breathing $\left(1069 \mathrm{~cm}^{-1}\right)$ and $v(\mathrm{CC})$ ring-stretching $\left(1575 \mathrm{~cm}^{-1}\right)$ modes, which appear very weak in the Raman spectrum of 4-MBA (figure 3a), are not only found to be remarkably enhanced in the obtained SERS spectrum of 4-MBA absorbed on the Ag/PU nanofibres (figure $3 b$ ), but also other intense peaks, $\delta(\mathrm{CH})$ bends $\left(1136\right.$ and $\left.1176 \mathrm{~cm}^{-1}\right)$, are observed. The high SERS performance of the present substrate can be explained by the electromagnetic field enhancement associated with surface-plasmon-coupled metal NPs, which have the appropriate interparticle gap, suitable for the generation of Raman 'hotspots'. While the EF of SERS substrates cannot be accurately calculated due to the rough surface of the $\mathrm{Ag} / \mathrm{PU}$ nanofibres, our rough estimation gave an $\mathrm{EF}$ value of $10^{5}$, as compared to similar $\mathrm{Ag} / \mathrm{PU}$ fibrous nanostructures reported previously. ${ }^{14}$ It is well accepted that the SERS enhancement is closely related to the nanostructures of the Ag NP aggregates on/in the polymeric nanofibres support. ${ }^{13,14}$ Besides, the distance of the probe to the metal surface has been found to play a dominant role in determining the final enhancement, as reported in an earlier study, ${ }^{22,23}$ and successful impregnation of Ag NPs onto the surface of

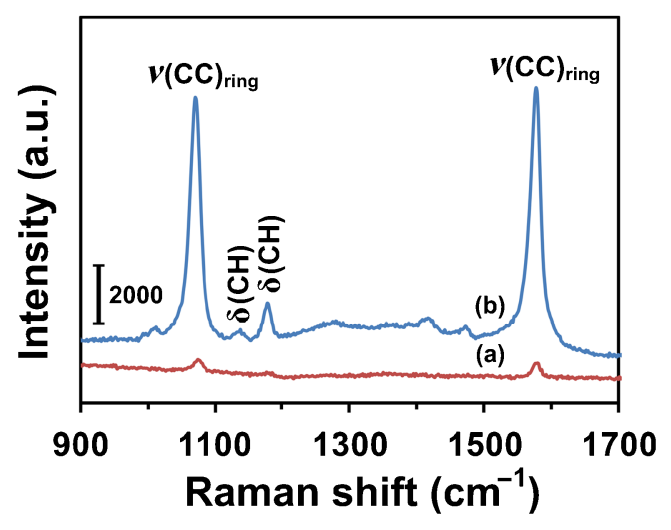

Figure 3. (a) Raman spectra of 4-MBA and (b) SERS spectra of 4-MBA absorbed on the Ag/PU nanofibres at a concentration of $10^{-4} \mathrm{M}$.

the PU nanofibres by simple polyol method could achieve direct contact between analyte molecules and silver. It is important that the mechanical robustness and flexibility of $\mathrm{Ag} / \mathrm{PU}$ nanofibre can be tailored into any shape, which would be extremely useful for mobile SERS applications. ${ }^{13,24}$

\section{Conclusion}

In summary, simultaneous synthesis and decoration of $\mathrm{Ag}$ NPs on the electrospun PU nanofibres that were made by electrospinning process was successfully performed via the polyol method without the need of any binding agent or surfactant except of hot ethylene glycol solution. Morphological analysis demonstrated that the Ag NPs with narrow size 
distribution were firmly attached to the fibre surface. Furthermore, SERS spectra showed comparatively high intensity in Raman characteristic peaks. Our present method is simple, safe and cost-effective, and the obtained composite materials may potentially a good candidate as SERS substrates.

\section{Acknowledgements}

Amarjargal acknowledges the support of a fund from Chonbuk National University Grant Fellow Project 2012 (Research Professor Program). This research was supported by a grant from the Basic Science Research Program through the National Research Foundation of Korea (NRF) funded by the Ministry of Education, Science and Technology (Project no. 2012-0001611) and also by a grant from the Regional Research and Development Cluster Project (B0009719) funded by the Ministry of Knowledge Economy of Korea. Partial funding was also provided by the Ministry of Education, Science and Technology through the Leaders in Industry-University Cooperation (LinC) Project (Project no. 2012-C-0043-010111). We also thank KBSIJeonju (Korea) for taking Raman measurement high-quality FESEM images.

\section{References}

1. Duan J L, Yang M, Lai Y C, Yuan J P and Zhan J H 2012 Anal. Chim. Acta $\mathbf{7 2 3} 88$

2. Jun B H, Noh M S, Kim J, Kim G, Kang H, Kim M S, Seo Y T, Baek J, Kim J H, Park J, Kim S, Kim Y K, Hyeon T, Cho M H, Jeong D H and Lee Y S 2010 Small 6119

3. Kneipp J, Kneipp H, Wittig B and Kneipp K 2010 Nanomed.Nanotechnol. Biol. Med. 6214

4. Li J M, Ma W F, Wei C, You L J, Guo J, Hu J and Wang C C 2011 Langmuir 2714539

5. Mulvihill M, Tao A, Benjauthrit K, Arnold J and Yang P 2008 Angew. Chem.-Int. Ed. 476456
6. Pienpinijtham P, Han X X, Ekgasit S and Ozaki Y 2011 Anal. Chem. 833655

7. Wang F L, Widejko R G, Yang Z Q, Nguyen K T, Chen H Y, Fernando L P, Christensen K A and Anker J N 2012 Anal. Chem. 848013

8. Naumann H, Klare J P, Engelhard M, Hildebrandt $\mathrm{P}$ and Murgida D H 2006 Photochem. Photobiol. Sci. 51103

9. Zhao J, Pinchuk A O, Mcmahon J M, Li S Z, Ausman L K, Atkinson A L and Schatz G C 2008 Acc. Chem. Res. 411710

10. Cao M H, Cheng S, Zhou X Z, Tao Z X, Yao J L and Fan L J 2012 J. Polym. Res. 199810

11. Liu S P, Chen N, Li L X, Pang F F, Chen Z Y and Wang T Y 2013 Opt. Mater. 35690

12. Tan E Z, Yin P G, You T T, Wang H and Guo L 2012 ACS Appl. Mater. Interfaces 43432

13. Zhang L F, Gong X, Bao Y, Zhao Y, Xu M, Jiang C Y and Fong H 2012b Langmuir 2814433

14. Bao Y, Lai C, Zhu Z, Fong H and Jiang C 2013 RSC Adv. 3 8998

15. Tijing L D, Park C-H, Choi W L, Ruelo M T G, Amarjargal A, Pant H R, Im I-T and Kim C S 2013 Composites Part B: Engineering 44613

16. Amarjargal A, Tijing L D, Park C-H, Im I-T and Kim C S 2013 Eur. Polym. J. 493796

17. Amarjargal A, Tijing L D, Ruelo M T G, Lee D H and Kim C S 2012 Mater. Chem. Phys. 135277

18. Dong H, Wang D, Sun G and Hinestroza J P 2008 Chem. Mater. 206627

19. Formo E, Lee E, Campbell D and Xia Y N 2008 Nano Lett. 8 668

20. Zhang P, Shao C L, Li X H, Zhang M Y, Zhang X, Sun Y Y and Liu Y C 2012c J. Hazard. Mater. 237331

21. Orendorff C J, Gole A, Sau T K and Murphy C J 2005 Anal. Chem. 773261

22. Michaels A M, Nirmal M and Brus L E 1999 J. Am. Chem. Soc. 1219932

23. Zhang C L, Lv K P, Cong H P and Yu S H 2012a Small 8648

24. He D, Hu B, Yao Q F, Wang K and Yu S H 2009 ACS Nano 3 3993 